\title{
N89-24557
}

\section{The structure of sheared turbulence near a plane boundary}

\author{
By MOON J. LEE $\nmid$ AND J. C. R. HUNT $\ddagger$
}

An analysis is presented of how a plane boundary affects the structure of turbulence in a sheared free stream. A uniform-shear boundary layer (USBL) is formulated with slip velocity condition at the surface, and inhomogeneous rapid distortion theory is applied. The effects of 'blocking' by the surface on the turbulence structure in USBL is compared with those in the shear-free boundary layer (SFBL).

Shear produces highly anisotropic eddies elongated in the flow direction. The spanwise spectra of the streamwise velocity $\Theta_{11}\left(\kappa_{3} ; y\right)$ suggest the existence of the streaky structures in the flow. The mean streak spacing estimated from the energy spectra increases with the distance from the surface, in qualitative agreement with previous measurements and computations.

The vertical velocity variance $\overline{v^{2}}$ is reduced with shear at all heights, roughly in proportion to the reduction in the homogeneous value, but the shape of the profile remains unchanged only near the surface: $\overline{v^{2}} /{\overline{v^{2}}}^{(H)} \sim y^{2 / 3}$. The turbulent shear stress $-\overline{u v}$ increases with total shear at all distances from the boundary. Scaled with the homogeneous value, the profile of the shear stress does not vary with time. The universal profile near the surface is $\overline{u v} / \overline{u v}^{(H)} \sim y^{2 / 3}$, similar to the vertical variance profile.

The streamwise integral scales increase with shear, indicating elongation of the streamwise extent of eddies. It is shown that, at given total shear, the spanwise extent of the streaks determined from $L_{u u}^{(z)}$ widens as the boundary is approached. The smallest of the integral scales, $L_{v v}^{(x)}$, is a measure of the dissipation scale, and decreases with shear in a self-similar way: $L_{v v}^{(z)} \sim y$.

\section{Introduction}

\subsection{Motivation and background}

In most models of sheared turbulent flows near plane boundaries, it is assumed that the mean 'shear rate' $S=d U / d y$ controls the structure of turbulence, especially the relation between the Reynolds shear stress $-\overline{u v}$ and the energy of the turbulence $q^{2}=\overline{u_{i} u_{i}}$. There is some support for this assumption when calculations for $\overline{u^{2}}$ and $-\overline{u v}$ and the cross-correlations $R_{i j}(r)$ in homogeneous uniform-shear flows $U=U_{0}+S y$ are compared with computations and measurements in turbulent boundary layers where $S$ is not uniform (Lee, Kim \& Moin 1987; Townsend 
1976). For close agreement between the two situations at a given height $y$, it was found necessary that the homogeneous shear and the boundary layer should have the same dimensionless shear rate $S^{*}=S L / q$ ( $L$ is an appropriate length scale), and that total shear $\beta=S t$ should be chosen appropriately. This homogeneousshear assumption is the basis of Reynolds-stress transport modelling as explained by Lumley (1978).

However, it is also known that the larger scales of motion (defined as greater than $y$ at a height $y$ ) are affected by the boundary, because vertical motions are blocked at $y=0$ (Bradshaw 1967; Townsend 1961). In turbulent flows, without shear near a rigid boundary or density interfaces, previous investigations (experimental, theoretical and computational) have shown that the blocking effect changes the variances of the different turbulence components $\overline{u^{2}}, \overline{v^{2}}, \overline{w^{2}}$, and the length scales. The largest effect is on the variance and the streamwise integral scale $L_{22}^{(x)}$ of the normal component $\overline{v^{2}}$; near the boundary, $\overline{v^{2}} \sim y^{2 / 3}$ and $L_{22}^{(x)} \sim y$ (Hunt \& Graham 1978; Hunt 1984).

Since there are many interesting and important turbulent flows near flat boundaries in which there are quite different shear rates, it is important to understand and quantify the effect of blocking by a surface in the presence of shear.

There have been previous unpublished studies using rapid distortion theory by Maxey (1978) and Durbin (1979).

Hunt, Moin, Moser \& Spalart (1987a) analysed the correlations between two points $y$ and $y_{0}\left(y \leq y_{0}\right)$ of the normal velocity component, $\widetilde{R}_{22}\left(y_{0}, y\right)$ using direct numerical simulations. It was found that the two-point correlation is, as hypothesised, a function of $y / y_{0}$, but the form differs from the linear profile in shear-free boundary layers (Hunt 1984), and is less than $y / y_{0}$ (figure 1 ).

Hunt, Spalart \& Mansour (1987b) examined the variation of the dissipation length $L_{\epsilon}$ defined as $\epsilon /{\overline{v^{2}}}^{3 / 2}$. They compared computations in the boundary layer and channel flow with a simple model $L_{\epsilon}^{-1}=A_{\mathrm{B}} / y+A_{\mathrm{S}}(d U / d y){\overline{v^{2}}}^{1 / 2}$ (Hunt, Stretch \& Britter 1988). The two terms represent the effect of blocking and shear, respectively. Good agreement was found except at the outer edge of the boundary layer. The sensitivity to shear of the dissipation scale is clearly much greater than for the crosscorrelations $\widetilde{R}_{22}$ and $\widetilde{R}_{12}$, which are dominated by blocking. An important aim of the present work is to explain this difference by looking closely at different length scales.

\subsection{Uniform-shear boundary layer}

It is possible to use Rapid Distortion Theory (RDT) to study how homogeneous turbulence in a uniform shear is deformed when a plate is suddenly introduced into the flow at time $t=0$. The analysis is inviscid so the only effect of the plate is to block velocity fluctuations normal to itself. In addition, the mean velocity profile is not changed. Because the mean vorticity remains uniform, this leads to a great simplification in the analysis. In reality, the presence of the wall leads to Reynolds stress gradients, which would over a long period affect the mean velocity profile; this is the effect we ignore here. 


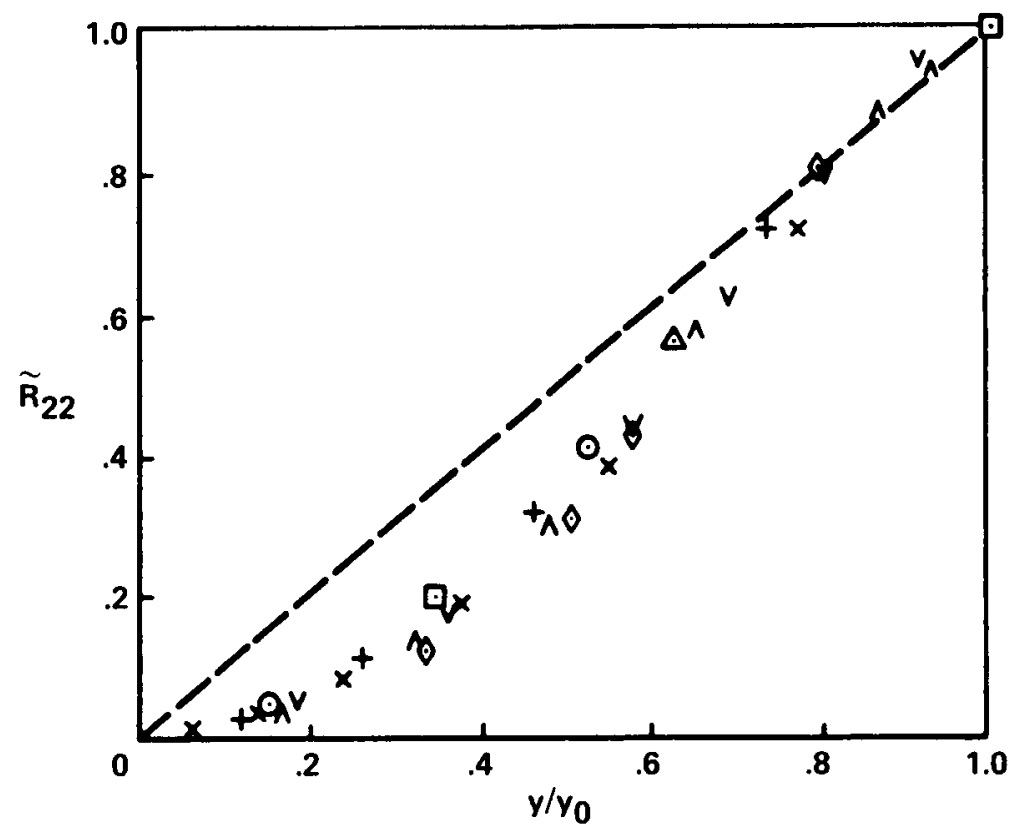

FIgure 1. Cross-correlation of $v$ at heights $y_{0}$ and $y$, normalized by $\overline{v^{2}}\left(y_{0}\right)$ computed from numerical simulations (turbulent boundary layer, Spalart 1987; plane channel flow, Kim, Moin \& Moser 1987). Symbols, data from numerical simulation; - - - , theoretical prediction and data for SFBL (Hunt 1984): $\widetilde{R}_{22} \simeq y / y_{0}$. [From Hunt et al. (1987a).]

The detailed aspect of the analysis that needs considering is the difference between the blocking effect of the boundary on the sheared turbulence compared with unsheared turbulence. In a shear-free boundary layer (SFBL), there is isotropic turbulence in the free stream, which leads to both horizontal components $\overline{u^{2}}$ and $\overline{w^{2}}$ increasing equally near the wall as a result of the irrotational fluctuation being axisymmetric about the $y$-axis. However, in a uniform-shear boundary layer (USBL), the turbulence in the free stream above the surface is quite anisotropic. This leads to a different redistribution of energy between $u$ and $w$ at the surface (Wong 1987) and a change in the interaction with the mean shear. This question has not been analysed in detail before.

The particular computations that are presented here are: (i) the variation with $y$ of the one-dimensional spectra $\Theta_{i j}\left(\kappa_{1} ; y\right)$ and $\Theta_{i j}\left(\kappa_{3} ; y\right)$ at different total shear $\beta=S t$; (ii) the Reynolds stresses $\overline{u_{i} u_{j}}$ as a function of $y$; and (iii) the variation with $y$ of the integral length scales in the homogeneous directions $(x, z)$ of the different velocity components $L_{i j}^{\left(x_{k}\right)}$. The smallest of these integral scales is usually a good indicator of the dissipation scale $L_{\epsilon}$, though this point has not been investigated systematically! 


\section{Analysis}

\subsection{Formulation of the problem}

We summarize here the assumptions, equations and boundary conditions governing the linearised (RDT) analysis of a turbulent velocity field $u(x, t)$ in a uniform shear flow $\mathbf{U}=\left(U_{0}+S y, 0,0\right)$ (see figure 2).

The condition for ignoring the nonlinear inertial terms over the period of the distortion is that the shear is strong enough for the straining terms to dominate over nonlinear inertial terms, that is

$$
S^{*}=S L / q \gg 1
$$

Over a period of time, the small nonlinear terms affect the redistribution of turbulence energy and momentum between different components. If $S^{*} \sim 1$, this relaxation time $t_{\mathrm{r}}$ is of order $L / q$, so the criterion for importance of nonlinear terms is that

$$
t>t_{\mathbf{r}} \sim L / q
$$

(Gartshore, Durbin \& Hunt 1983; Bertoglio 1986).

But if $S^{*}$ is large, the rate at which the anisotropy is growing by linear processes is so much greater than by nonlinear processes that, as Lee et al. (1987) have shown, the nonlinear redistributive processes are negligible (especially for $\overline{u^{2}}$ and $\overline{u v}$ ) even when $t \geq t_{\mathrm{r}}$.

For the linearised analysis of the USBL, it is also necessary to assume that the gradient of the Reynolds stress has a weak effect on the mean velocity profile over the time $T_{\mathrm{D}}$ of distortion:

$$
T_{\mathrm{D}}\left|-\frac{\partial^{2} \overline{u v}}{\partial y^{2}}\right| \ll S
$$

or

$$
S^{*}=S L / q \gg q T_{\mathrm{D}} / L \text {. }
$$

The inviscid analysis used here can only correspond to physical experiments involving a real viscous fluid, if the viscous stresses are negligible within the flow for energy-containing eddies and if the no-slip boundary condition can be neglected (see figure 2). Hunt \& Graham's (1978) detailed analysis of both these effects led to the following conditions for neglecting them:

$$
\begin{gathered}
q L / \nu \gg 1, \\
\sqrt{\nu T_{\mathrm{D}}} \ll L \quad \text { and } \quad \delta_{\mathrm{v}} \ll L,
\end{gathered}
$$

where $\delta_{v}$ is the thickness of the viscous layer on the surface, which must exist if the velocity $U_{0}$ of the free stream at $y=0$ is not equal to the velocity of the surface $U_{\mathrm{b}}$ (figure 2b-i). If $U_{\mathrm{b}}=U_{0}$, as in the moving-belt experiment of Uzkan \& Reynolds (1967) and Thomas \& Hancock (1977), there is no mean boundary layer, but the 
(a)

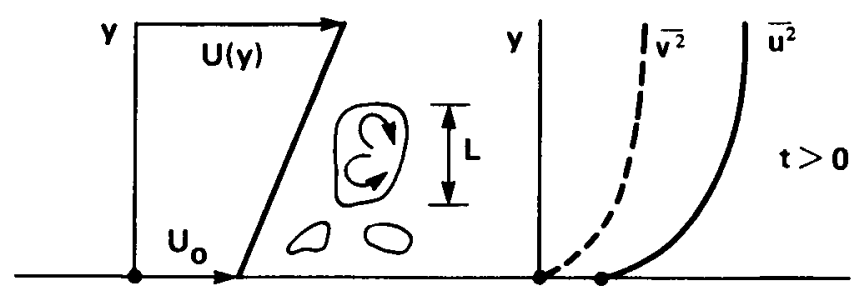

(b) i)
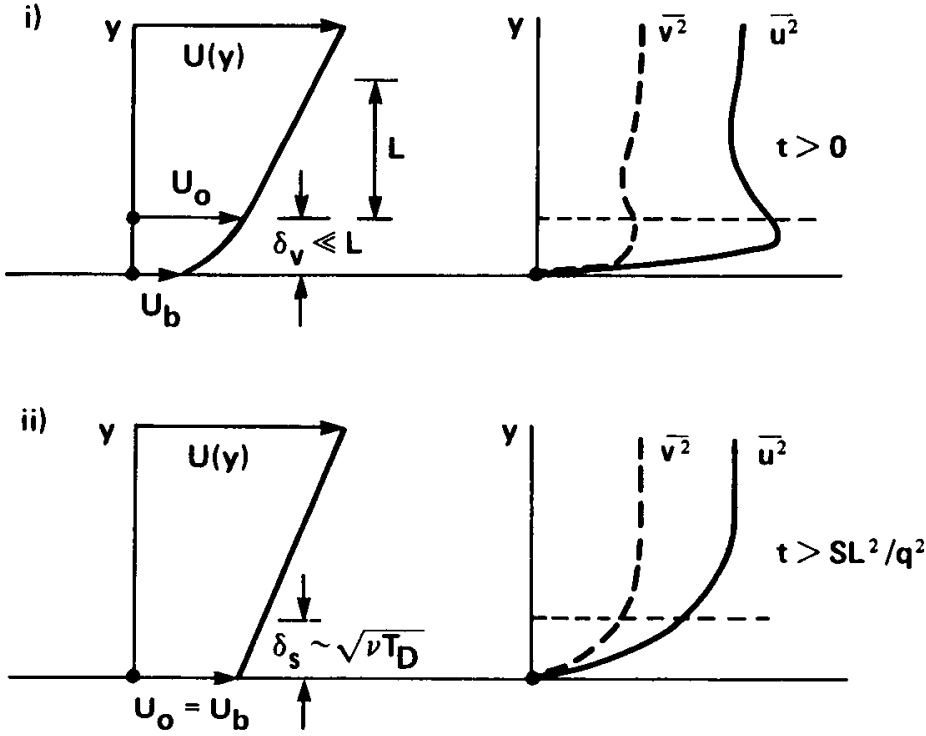

(c)

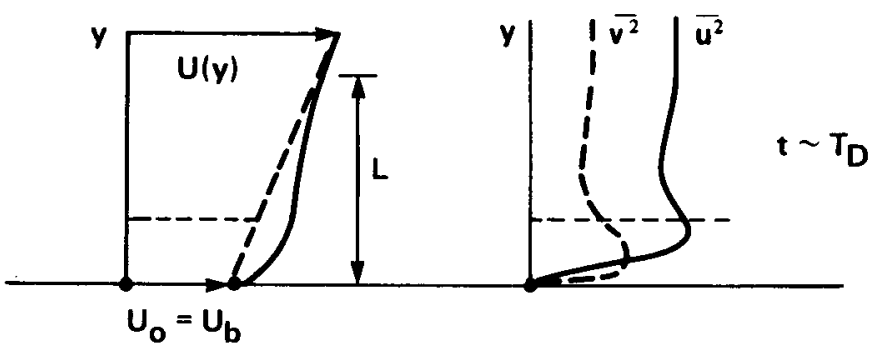

FIgURE 2. Schematic diagram of a uniform-shear boundary layer (USBL), showing (a) the ideal problem; (b) the experimental reality: (i) if $U_{\mathbf{b}} \neq U_{0}$, theory requires $\delta_{\mathrm{v}} \ll L$, (ii) if $U_{\mathrm{b}}=U_{0}$, theory requires $\delta_{\mathrm{s}} \sim \sqrt{\nu T_{\mathrm{D}}} \ll L ;(c)$ the slow distortion of the mean velocity profile $\left(t \sim T_{\mathrm{D}}\right)$ because of the Reynolds-shear-stress gradient. 
fluctuating velocity must still be zero. This thin surface layer affected by this no-slip fluctuating condition is of order $\sqrt{\nu T_{\mathbf{D}}}$ (figure $2 b$-ii).

Given the assumptions (2.1) to (2.5), the equations for the linearised problem are

$$
\begin{gathered}
\frac{\bar{D} \mathbf{u}}{D t}=-\nabla p-S v \mathbf{i}, \\
\nabla \cdot \mathbf{u}=0,
\end{gathered}
$$

where $\bar{D} / D t=\partial / \partial t+U \partial / \partial x, \mathrm{U}=\left(U_{0}+S y, 0,0\right)$ and $p$ is the kinematic pressure fluctuation. The initial and boundary conditions are given by

$$
u(x, t)=u^{(H)}(x, t) \text { at } t=0,
$$

where $u^{(B)}$ is a prescribed random velocity field, and

$$
v(x, y=0, z, t)=0 \text { for } t>0 .
$$

The analytical solution is obtained as a sum of the homogeneous flow field, $\left\{\mathbf{u}^{(\boldsymbol{H})}, \boldsymbol{p}^{(\boldsymbol{B})}\right\}(\boldsymbol{x}, t)$, subject to uniform shear, and the blocking flow field induced by the surface, $\left\{\mathbf{u}^{(\mathrm{B})}, p^{(\mathrm{B})}\right\}(\mathbf{x}, t)$ :

$$
\begin{aligned}
& \mathbf{u}(\mathbf{x}, t)=\mathbf{u}^{(\mathbf{B})}(\mathbf{x}, t)+\mathbf{u}^{(\mathbf{B})}(\mathbf{x}, t), \\
& p(\mathbf{x}, t)=p^{(\mathbf{B})}(\mathbf{x}, t)+p^{(\mathbf{B})}(\mathbf{x}, t) .
\end{aligned}
$$

Then, $u^{(H)}(x, t)$ satisfies $(2.6)$ subject to $(2.8 a)$ at $t=0$; it does not satisfy (2.8b). But $u^{(B)}(x, t)$ satisfies (2.6) subject to

$$
v^{(\mathbf{B})}(\mathbf{x}, t)=0 \quad \text { at } \quad t=0 .
$$

From (2.8) and (2.9), it can be shown that

$$
v^{(B)}(x, y=0, z, t)=-v^{(\mathrm{H})}(x, y=0, z, t) \text { for } t>0,
$$

and

$$
\mathbf{u}^{(\boldsymbol{B})}(\mathbf{x}, t) \rightarrow 0 \text { as } y \rightarrow \infty .
$$

By taking the curl of (2.6), it follows that

$$
\frac{\bar{D}}{D t} \nabla^{2} v^{(B)}=0 \text {. }
$$

So from (2.10a), one finds that this equation for $v^{(B)}(x, t)$ reduces to Laplace's equation: $\nabla^{2} v^{(B)}=0$. Once $v^{(B)}(x, t)$ is obtained, $p^{(B)}(x, t)$ is obtained from

$$
\nabla^{2} p^{(B)}=-2 S \frac{\partial v^{(B)}}{\partial x} \text {. }
$$

Thence from (2.6), $w^{(B)}(x, t)$ and $u^{(B)}(x, t)$ are calculated.

The statistical and Fourier analyses are similar to the method for inhomogeneous RDT developed by Hunt (1973), and used for SFBL by Hunt \& Graham (1978) and for a uniform-shear layer by Gartshore, Durbin \& Hunt (1983). 


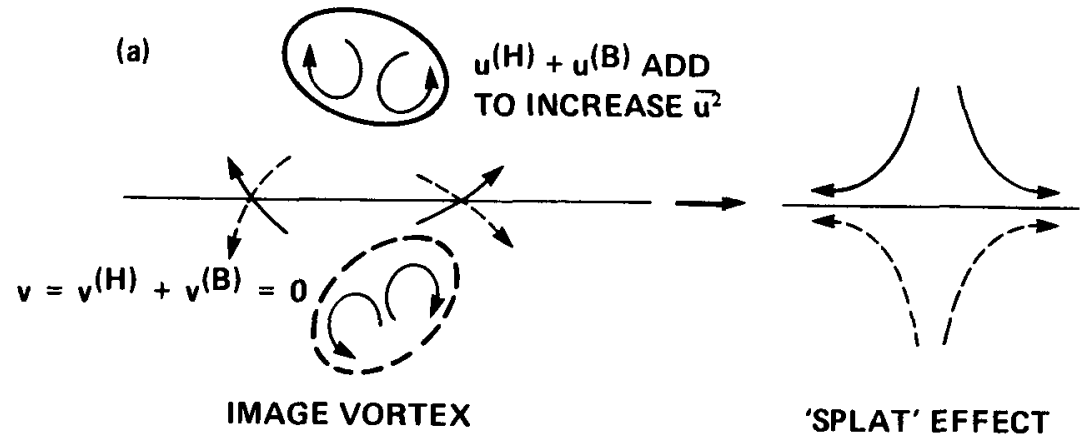

(b)

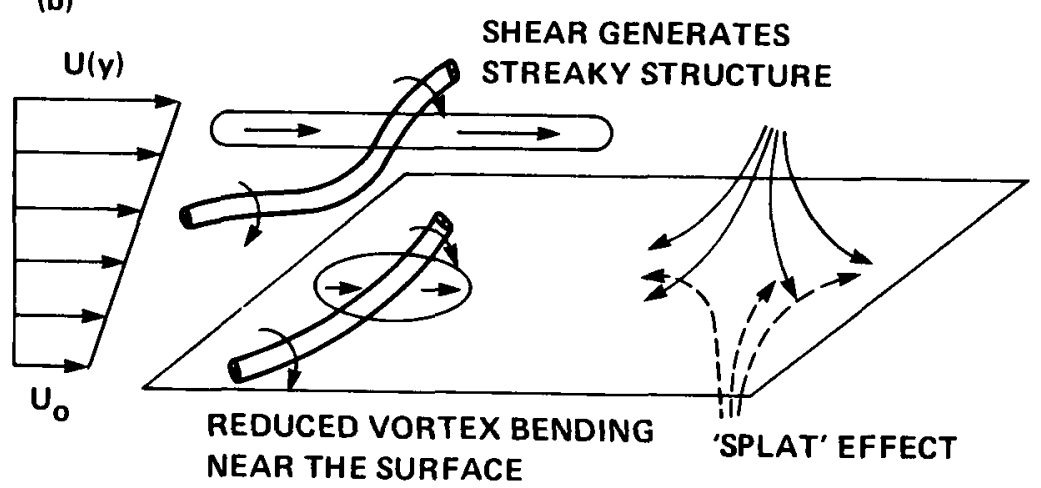

FIGURE 3. Schematic diagram to show difference between the mechanisms for shearfree boundary layer (SFBL) and for uniform-shear boundary layer (USBL): (a) SFBL, image vortex below $y=0$ induces irrotational velocity $\mathbf{u}^{\left({ }^{(B)}\right.}$ above $y=0 ;(b)$ USBL, reduced vortex bending near the surface reduces $\overline{u^{2}}$ while the splat effect increases $\overline{u^{2}}$.

\subsection{Physical interpretation}

The difference in their mechanics between a shear-free boundary layer (SFBL) and a uniform-shear boundary layer (USBL) can be understood from the linearised vorticity equation obtained by taking the curl of (2.6):

$$
\frac{\bar{D} \boldsymbol{\omega}}{D t}=(\boldsymbol{\omega} \cdot \nabla) \mathbf{U}+(\Omega \cdot \nabla) \mathbf{u} .
$$

In a SFBL where $\nabla \mathbf{U}=\mathbf{0}$ and $\boldsymbol{\Omega}=\mathbf{0}$, this equation reduces to

$$
\bar{D} \omega
$$

The effect of the boundary is simply to introduce an irrotational velocity field, so the vorticity is not affected by the boundary, i.e.

$$
\mathbf{u}^{(\mathbf{B})}(\mathbf{x}, t)=\nabla \phi(\mathbf{x}, t), \quad \boldsymbol{\omega}(\mathbf{x}, t)=\omega^{(\mathbf{H})}(\mathbf{x}, t) .
$$


This irrotational velocity field induced by image vorticity below $y=0$ produces the splat effect: the vertical component $v^{(B)}$ blocks $v^{(H)}$ so that $v=0$ at $y=0$, while the horizontal components add to increase $\overline{u^{2}}$ and $\overline{w^{2}}$ (figure $3 a$ ).

But in a USBL the addition of the blocking velocity field $u^{(B)}(x, t)$ affects $\omega$ because of the term $(\Omega \cdot \nabla) \mathbf{u}$, which is the effect of velocity perturbations distorting the vorticity $\Omega=\left(0,0, \Omega_{z}\right)$ of the mean shear. The terms $\Omega_{z} \partial v / \partial z$ and $\Omega_{z} \partial w / \partial z$ primarily determine $\omega_{y}$ and $\omega_{z}$, and $u$ has contributions from $\Omega_{z} \int_{0}^{z} \omega_{y} d z \sim \Omega_{z} v$ and $\Omega_{z} \int_{0}^{y} \omega_{z} d y \sim \Omega_{z} \int \partial v / \partial y d y \sim \Omega_{z} v$. (Note that in a shear flow the eddies are elongated in the flow direction, so $\partial u / \partial x \ll \partial v / \partial y$.) Thus, near the boundary, as $v$ decreases, $\overline{u^{2}}$ and $-\overline{u v}$ also decreases. However, in the USBL, there is also a tendency of $\overline{u^{2}}$ and $\overline{w^{2}}$ to increase because of the impacting (or splatting) of the normal fluctuations at the boundary. The analysis shows the different contributions by these two mechanisms.

\section{Results and discussion}

We assume here that the initially homogeneous turbulence is isotropic and has the von Kármán spectrum of the dimensionless form

$$
E^{(\mathrm{H})}(\kappa)=\frac{A \kappa^{4}}{\left(c_{\mathrm{K}}+\kappa^{2}\right)^{2+\mu}}
$$

where $\mu=\frac{5}{6}, A=\frac{55}{9 \pi} c_{\mathrm{K}}^{\mu}$ and $c_{\mathrm{K}}=0.558$. Note that this spectrum behaves as $\kappa^{4}$ in low-wavenumber region $(\kappa \ll 1)$ and has the $\kappa^{-5 / 3}$ Kolmorogov form at high wavenumbers $(\kappa \gg 1)$. All the quantities are made dimensionless by the initial r.m.s. velocity $u_{0}$ and integral length scale $L_{0}$. Note that in USBL the turbulence quantities change with total shear $\beta=S t$. In order to highlight the effects of shear on turbulence structure near a boundary, comparison is made with the results for SFBL $(\beta=0)$ (for details, see Hunt \& Graham 1978; Hunt 1984).

\subsection{Energy spectra}

In figure 4 , the one-dimensional energy spectra $\Theta_{i j}\left(\kappa_{1} ; y\right)$ are plotted for SFBL. When the turbulence is unsheared, the near-wall behavior of the spectra is such that there is no variation in $\Theta_{11}\left(\kappa_{1} \rightarrow 0 ; y\right)$, but the increase in $\Theta_{33}\left(\kappa_{1} \rightarrow 0 ; y\right)$ exactly balances the reduction in $\Theta_{22}\left(\kappa_{1} \rightarrow 0 ; y\right)$, viz.

$$
\begin{gathered}
\Theta_{11}^{\mathrm{SFBL}}\left(\kappa_{1} \rightarrow 0 ; y\right)=\Theta_{11}^{(\mathrm{H})}\left(\kappa_{1} \rightarrow 0\right)=1 / \pi, \\
\Theta_{22}^{\mathrm{SFBL}}\left(\kappa_{1} \rightarrow 0 ; y \rightarrow 0\right)+\Theta_{33}^{\mathrm{SFBL}}\left(\kappa_{1} \rightarrow 0 ; y \rightarrow 0\right)=1 / \pi,
\end{gathered}
$$

where $\Theta_{i j}^{(H)}\left(\kappa_{1}\right)$ are the one-dimensional energy spectra of the initially homogeneous, isotropic turbulence, i.e. $\Theta_{i j}\left(\kappa_{1} ; y \rightarrow \infty, t=0\right)$. Similar results can be found by a symmetry consideration: $\Theta_{33}^{\mathrm{SFBL}}\left(\kappa_{3} \rightarrow 0 ; y\right)=\Theta_{33}^{(\mathrm{H})}\left(\kappa_{3} \rightarrow 0\right)=1 / \pi, \Theta_{11}^{\mathrm{SPBL}}\left(\kappa_{3} \rightarrow\right.$ $0 ; y \rightarrow 0)+\Theta_{22}^{\mathrm{SBL}}\left(\kappa_{3} \rightarrow 0 ; y \rightarrow 0\right)=1 / \pi$. Asymptotic analysis (Hunt \& Graham 1978) shows that near the surface

$$
\Theta_{22}^{S F B L}\left(\kappa_{1} \rightarrow 0 ; y \rightarrow 0\right)=\Theta_{22}^{S F B L}\left(\kappa_{3} \rightarrow 0 ; y \rightarrow 0\right) \sim y^{5 / 3}
$$


The structure of sheared turbulence near a plane boundary

229
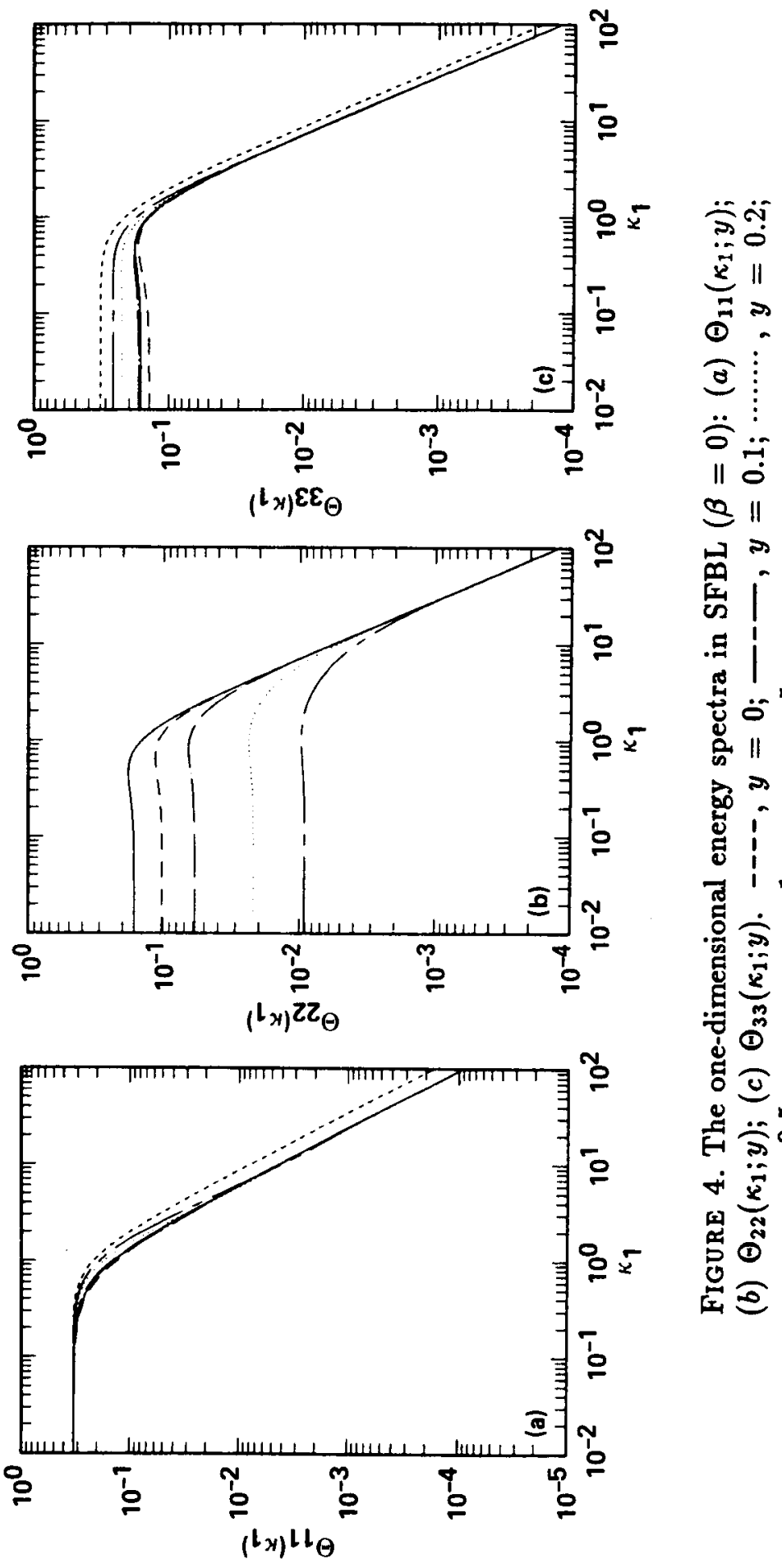

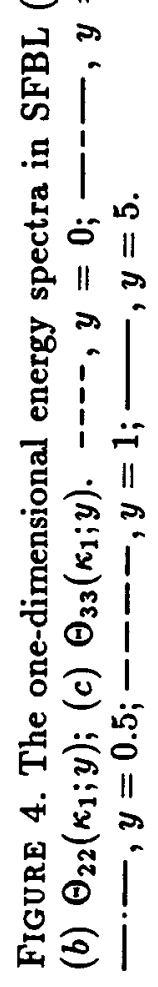


230

M. J. Lee and J. C. R. Hunt
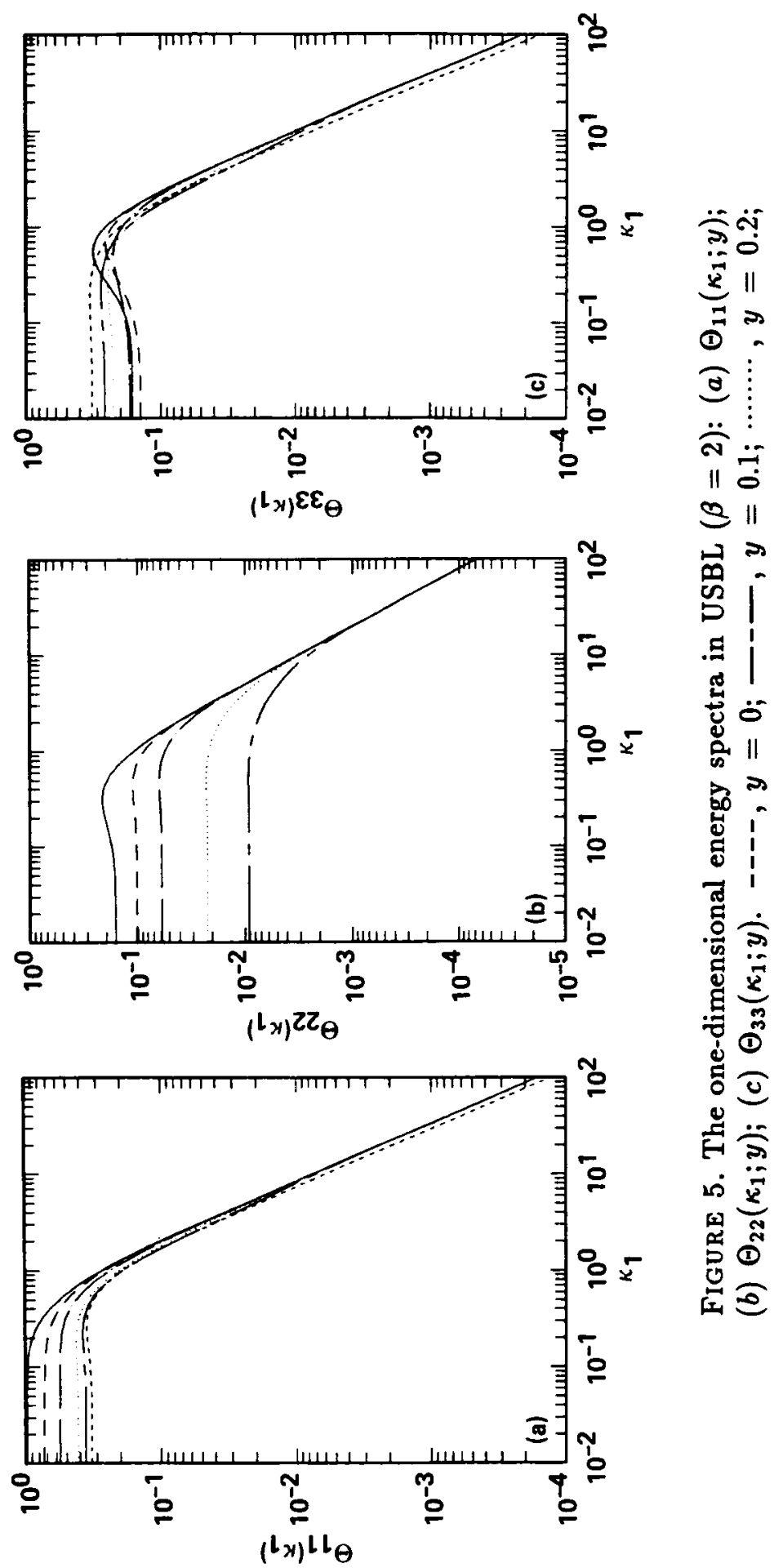
In order to examine the effect of shear on the energy spectra, the results for USBL (when $\beta=2$ ) are shown in figure 5 . The most salient feature is that, as $\kappa_{1} \rightarrow 0$, there is a dramatic increase with shear in the spectrum $\Theta_{11}\left(\kappa_{1} ; y\right)$ of the streamwise velocity at distances away from the surface (cf. figures $4 a$ and $5 a$ ). However, near the surface the spectrum is not affected by shear

$$
\Theta_{11}^{\text {USBL }}\left(\kappa_{1} \rightarrow 0 ; y \rightarrow 0\right)=\Theta_{11}^{\text {SFBL }}\left(\kappa_{1} \rightarrow 0 ; y\right)=1 / \pi \text {. }
$$

That is, very close to the surface, the streamwise motion of large-scale eddies is dominated by the induced image vortices as shown in figure 3(a), independent of shearing in the free stream. In contrast, shear does not change the values of $\Theta_{22}\left(\kappa_{1} \rightarrow 0 ; y\right)$ and $\Theta_{33}\left(\kappa_{1} \rightarrow 0 ; y\right)$ for all $y$ :

$$
\begin{aligned}
& \Theta_{22}^{\mathrm{USBL}}\left(\kappa_{1} \rightarrow 0 ; y\right)=\Theta_{22}^{\mathrm{SPBL}}\left(\kappa_{1} \rightarrow 0 ; y\right), \\
& \Theta_{33}^{\mathrm{USBL}}\left(\kappa_{1} \rightarrow 0 ; y\right)=\Theta_{33}^{\mathrm{SPBL}}\left(\kappa_{1} \rightarrow 0 ; y\right) .
\end{aligned}
$$

At high wavenumbers, however, the spectrum $\Theta_{22}\left(\kappa_{1} ; y\right)$ of the component normal to the boundary is significantly reduced by shear for all $y$ (cf. figures $4 b$ and $5 b$ ), which implies that the vertical motion of small-scale eddies is sensitive to shear. Interestingly enough, $\Theta_{11}\left(\kappa_{1} ; y \rightarrow 0\right)$ is reduced much more in the high-wavenumber region $\left(\kappa_{1}>1\right)$ than it is enhanced at low wavenumbers $\left(\kappa_{1}<1\right)$; in fact, $\overline{u^{2}}$ on the surface in the USBL is lower than the SFBL value: $\overline{u^{2}}(y=0)<\frac{3}{2}$.

It is of fundamental importance to observe the peaks in the spanwise spectrum $\Theta_{11}\left(\kappa_{3} ; y\right)$ of the streamwise velocity (figure 6a), which implies the existence of eddies at the corresponding spanwise scales. The peaks in $\Theta_{11}\left(\kappa_{3} ; y\right)$ at distances away from the wall and the high streamwise velocity variance (see figure 9 ) are direct evidence of the presence of streaky structures in the flow (see Kline et al. 1967). Notice that the peaks are much more discernible in the spectra at heights away from the wall, consistent with the earlier observation of the low- and high-speed streaks in homogeneous shear flow where there is no boundary (Lee et al. 1987). The fact that the streaks exist in sheared turbulence, independent of the presence of the boundary, but not in the SFBL strongly supports the assertion put forward by Lee et al. that the main mechanism of generating the streaks is the mean shear but not the wall blocking. This is supported also by the experiment of Uzkan \& Reynolds (1967).

As shear increases, the peak in the spectrum (or streaks) is discernible at heights closer to the wall (e.g. for $\beta=4$, see figure $6 b$ ). This means that, as shear is prolonged, the lateral extent of the streaks are mainly controlled by the effect of shear rather than by the blocking effect. Note that other aspects of the turbulence (e.g. $\overline{v^{2}}$ and $-\bar{u} \bar{v}$ ) remain affected by the blocking whatever the total shear.

It is of interest to examine how the mean spacing $\lambda_{z}$ between the streaks varies with the distance from the surface. The mean streak spacing is determined from the wavenumber $\kappa_{3}^{(s)}$ at which $\Theta_{11}\left(\kappa_{3} ; y\right)$ peaks, i.e. $\lambda_{z}=1 / \kappa_{3}^{(s)}$. It is clear from figure 7 that $\lambda_{z}$ increases with $y$, in qualitative agreement with measurements and computations in turbulent boundary layers (Kline et al. 1967; Kim et al. 1987). 

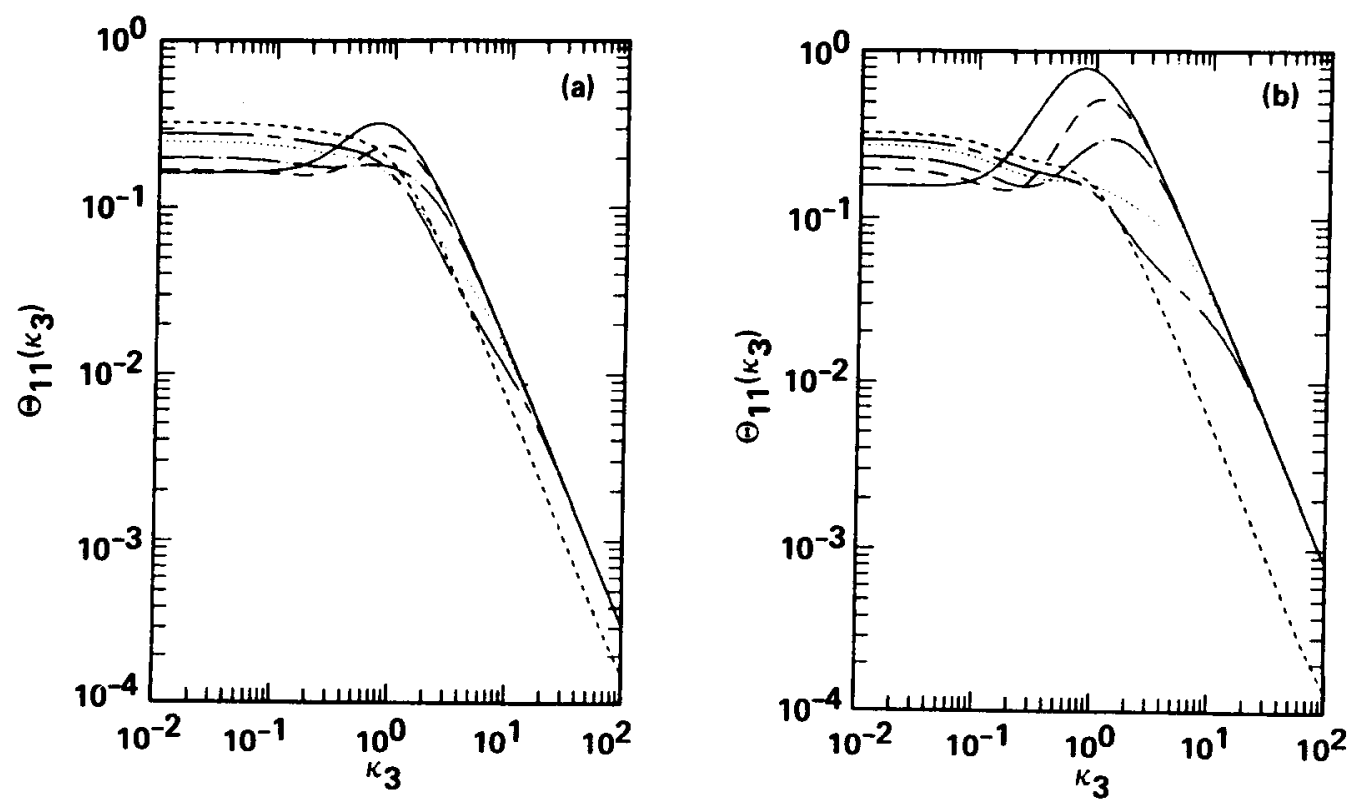

FIgURE 6. The one-dimensional energy spectra $\Theta_{11}\left(\kappa_{3} ; y\right)$ in USBL: $(a) \beta=2 ;(b)$ $\beta=4 .---, y=0 ;---, y=0.1 ; \cdots \cdots \cdots, y=0.2 ;-\cdot-, y=0.5 ;----$, $y=1 ;-, y=5$.

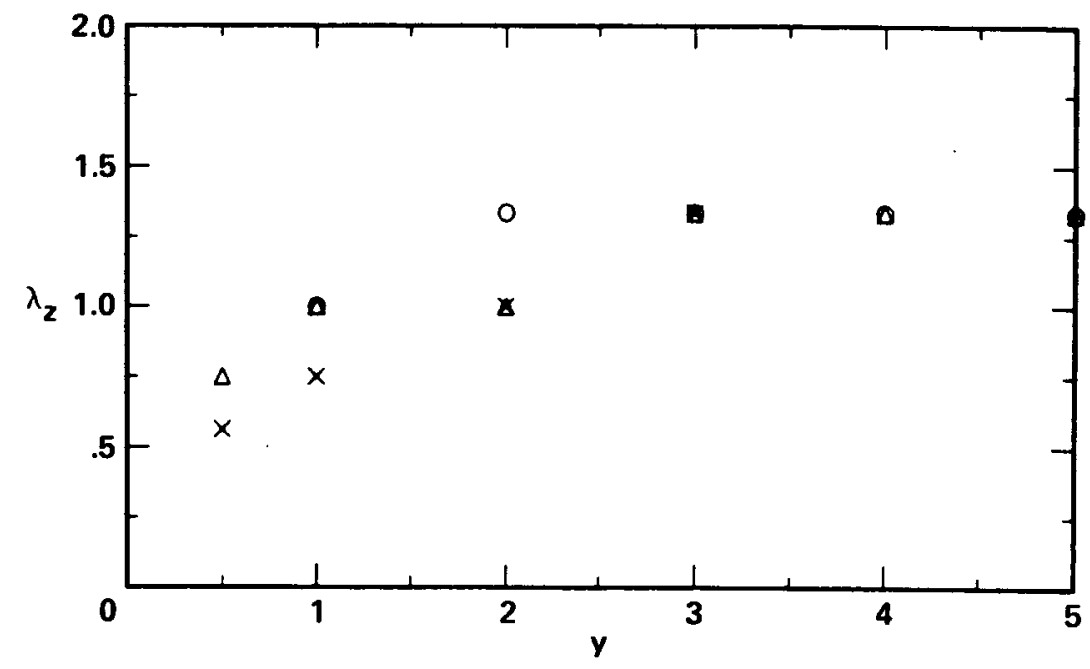

FIGURE 7. Variation with the distance from the boundary of the mean streak spacing, $\lambda_{z}$, determined from the wavenumber at which $\Theta_{11}\left(\kappa_{3} ; y\right)$ peaks. $O, \beta=2$; $\triangle, \beta=4 ; \times, \beta=8$. 


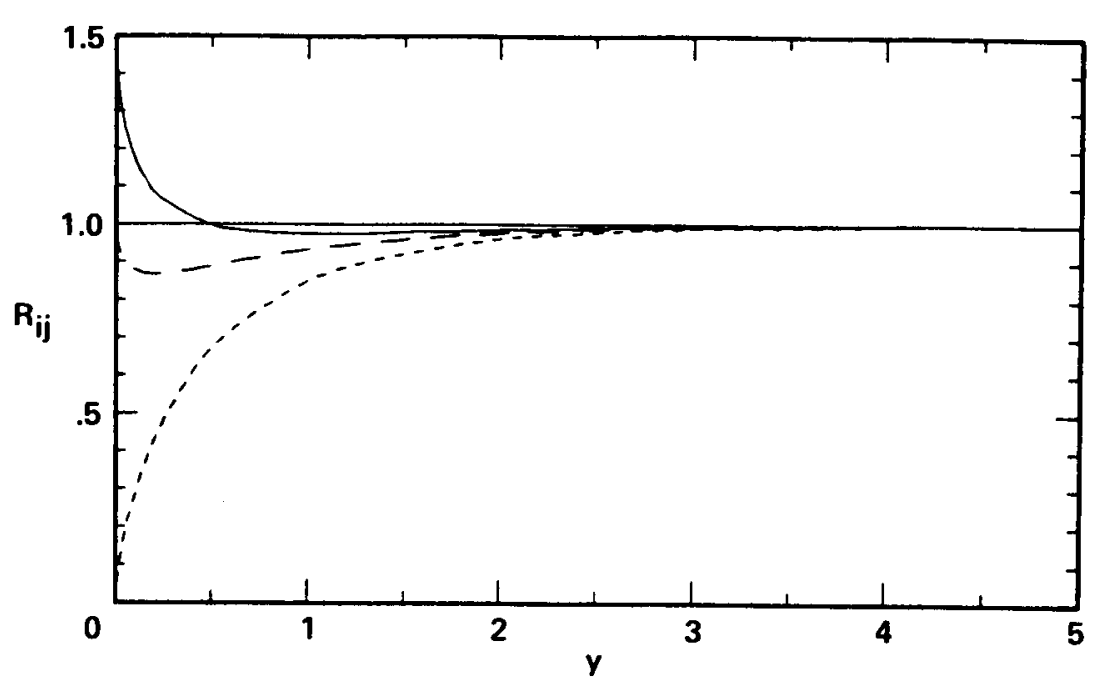

FIGURE 8. Profile of the Reynolds stresses in SFBL $(\beta=0)$. - - - , $\frac{1}{3} \overline{u_{i} u_{i}}$; $-\overline{u^{2}}=\overline{w^{2}} ;----, \overline{v^{2}}$.

The spanwise spectrum $\Theta_{33}\left(\kappa_{3} ; y\right)$ of the spanwise velocity fluctuation as $\kappa_{3} \rightarrow 0$ is unchanged for all $y$ :

$$
\Theta_{33}^{\text {USBL }}\left(\kappa_{3} \rightarrow 0 ; y\right)=\Theta_{33}^{\text {SFBL }}\left(\kappa_{3} \rightarrow 0 ; y\right)=\Theta_{33}^{(\text {H) }}\left(\kappa_{3} \rightarrow 0\right)=1 / \pi .
$$

Thus, (3.7) and (3.6b) indicate that in this flow the large-scale spanwise fluctuation is not affected by shear (cf. figs. $4 c$ and $5 c$ ). Also note that

$$
\Theta_{11}^{\text {USBL }}\left(\kappa_{3} \rightarrow 0 ; y \rightarrow 0\right)=\Theta_{11}^{\text {SPBL }}\left(\kappa_{3} \rightarrow 0 ; y \rightarrow 0\right)=1 / \pi \text {. }
$$

\subsection{Reynolds stresses}

In figure 8 , the Reynolds stresses $\overline{u_{i} u_{j}}$ are shown as functions of $y$ for SFBL. In SFBL, if the homogeneous field is isotropic, the horizontal variances are equal, $\overline{u^{2}}=\overline{w^{2}}$, for all $y$. Near the surface, the splat effect results in transfer of energy from the vertical component to the horizontal components:

$$
\left.\begin{array}{l}
\overline{u^{2}}(y \rightarrow 0)=\overline{w^{2}}(y \rightarrow 0)=\frac{3}{2}-O\left(y^{2 / 3}\right), \\
\overline{v^{2}}(y \rightarrow 0) \sim y^{2 / 3}
\end{array}\right\} \quad \text { in SFBL. }
$$

The horizontal variances are decreased from the wall value to the homogeneous value of unity within about $y=0.5$ from the wall. The vertical variance $\overline{v^{2}}$ shows 


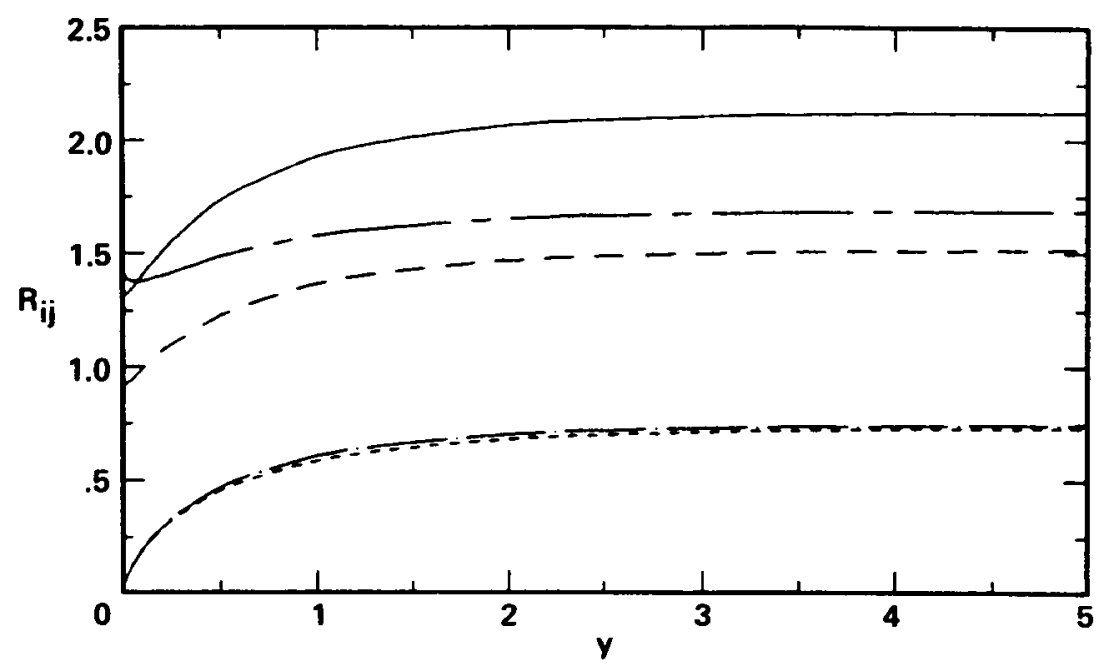

FIgURE 9. Profile of the Reynolds stresses in USBL $(\beta=2)$. - - - , $\frac{1}{3} \overline{u_{i} u_{i}}$; $\longrightarrow, \overline{u^{2}} ;---, \overline{v^{2}} ;---, \overline{w^{2}} ;-.-, \overline{u v}$.

monotonic increase from zero at the wall to $95 \%$ of its homogeneous value (unity) over a distance of about 2.

Variation of the kinetic energy in SFBL is not monotonic. Except at the surface where $q^{2} / q_{0}^{2}=1$, the kinetic energy is less than its homogeneous value; the ratio $q^{2} / q_{0}^{2}$ attains its minimum value of about 0.85 at $y \simeq 0.2$.

Figure 9 shows the profile of the Reynolds stresses in USBL $(\beta=2)$. An important aspect of USBL is the increase of the turbulent kinetic energy by the transfer from the mean flow to turbulence (i.e. turbulence production). Therefore, the kinetic energy is much larger in USBL than in SFBL (except in the vicinity of the surface), e.g. $50 \%$ increase in the homogeneous value when $\beta=2$ (cf. figues 8 and 9 ). The dip near the surface (at $y \simeq 0.2$ when $\beta=2$ ) is a direct consequence of the reduction of the horizontal variances there, as explained below. Note that in USBL the turbulent kinetic energy at the surface is not equal to the homogeneous value, unlike in SFBL.

In USBL the horizontal variances $\overline{u^{2}}$ and $\overline{w^{2}}$ are markedly enhanced by shear except very close to the wall. The reduction of these variances can be explained by the effect of the reduced bending of vortex filaments (see figure $3 b$ ); the spanwise variance $\overline{w^{2}}$ is less reduced than the streamwise variance $\overline{u^{2}}$.

Comparison of the vertical variance $\overline{v^{2}}$ in figures 8 and 9 shows that there is a substantial reduction with shear at all heights. [The apparently coincidental profiles of $\overline{v^{2}}$ and $-\overline{u v}$ in the figure 9 is incidental at this particular time $(\beta=2)$.] It is interesting to consider how $\overline{v^{2}}$ in the vicinity of the boundary changes with shear. 


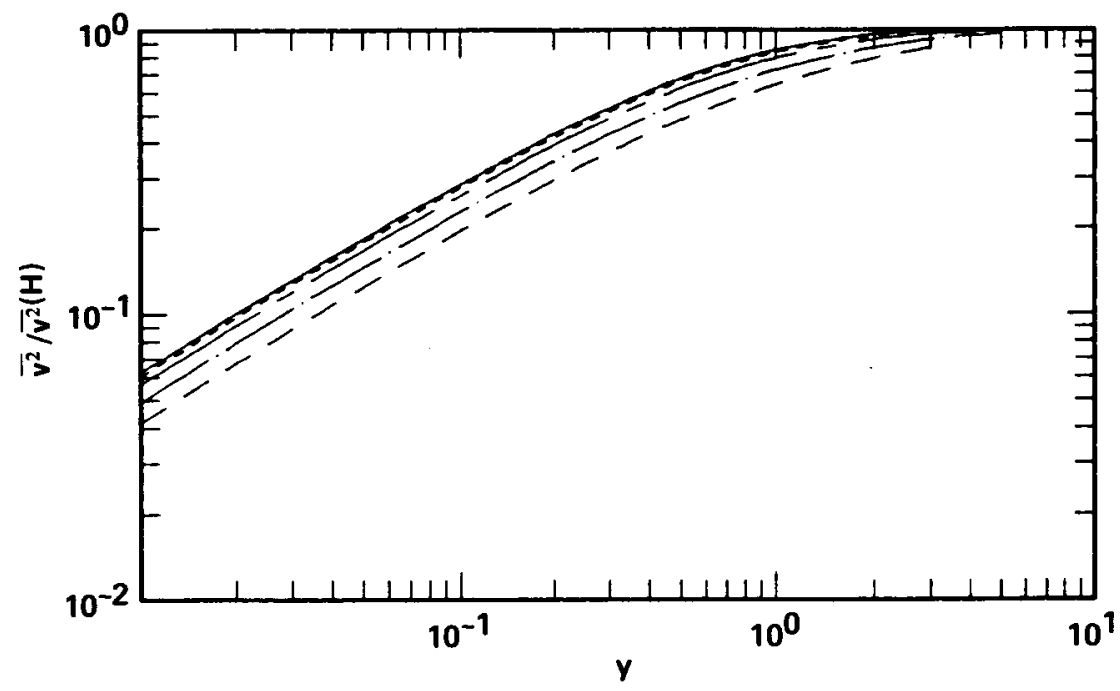

FIGURE 10. Variation of the vertical variance profile with total shear: $\overline{v^{2}} \sqrt{v^{2}}{ }^{(H)}$ vs. y.,$- \beta=0 ;-\cdots, \beta=1 ;--\longrightarrow, \beta=2 ;-.-, \beta=4 ;----, \beta=8$.

When scaled by the homogeneous value ${\overline{v^{2}}}^{(\boldsymbol{H})}$, the profiles of $\overline{v^{2}}$ at different values of $\beta$ show (figure 10) that as $y \rightarrow 0$

$$
\overrightarrow{v^{2}}(y \rightarrow 0, \beta) \sim{\overline{v^{2}}}^{(\not)}(\beta) y^{2 / 3} \quad \text { in USBL. }
$$

Another important difference in USBL is the development of the turbulent shear stress $-\overline{u v}$ with shear. Note that the shear stress is also sub ject to reduction by the wall blocking. The profile of the shear stress exhibits a monotonic increase from zero at the wall towards the homogeneous value that increases with shear. Thus, the same scaling used for $\overline{v^{2}}$ is used for the shear stress: as $y \rightarrow 0$,

$$
\overline{u v}(y \rightarrow 0, \beta) \sim \overline{u v}^{(\mathrm{H})}(\beta) y^{2 / 3} \quad \text { in USBL. }
$$

Figure 11 shows $\overline{u v} / \overline{u v}^{(H)}$ as a function of $y$ at different times. This function does not change with total shear. It is within $20 \%$ of the value of $\overline{v^{2}} / \overline{v^{2}}{ }^{(H)}$ at $\beta=0$.

\subsection{Integral length scales}

Integral length scales in the $x_{m}$-direction of the correlations between the velocity components $u_{i}$ and $u_{j}$ at $y$ are defined as

$$
L_{i j}^{\left(x_{m}\right)}(y)=\frac{\pi \Theta_{i j}\left(\kappa_{m}=0 ; y\right)}{\overline{u_{i} u_{j}}(y)} \quad(\text { no sum on } i \text { and } j) .
$$




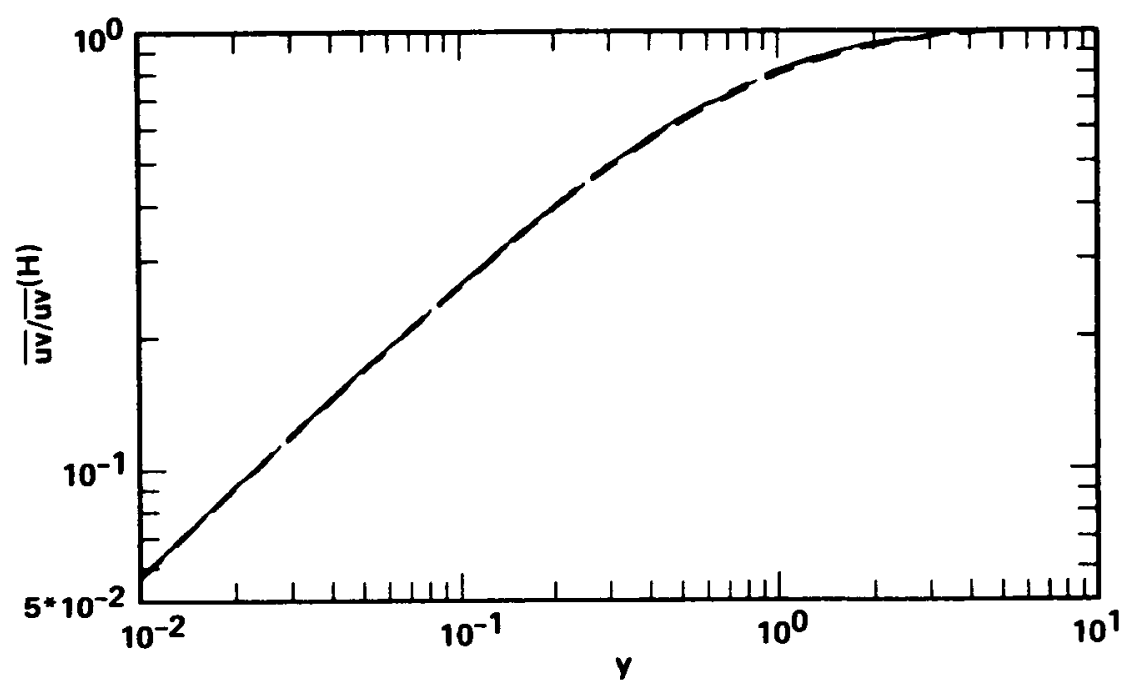

FIGURE 11. Variation of the turbulent shear-stress profile with total shear: $\overline{u v} / \overline{u v}(\mathbf{H})$ vs. $y$. ----, $\beta=1 ;---, \beta=2 ;--, \beta=4 ;----, \beta=8$.

Profiles of the streamwise integral scales $L_{u u}^{(x)}, L_{v v}^{(x)}$ and $L_{w w}^{(x)}$ in SFBL are shown in figure 12. Very close to the wall, the streamwise scale of the vertical velocity grows faster than that of the streamwise velocity; from (3.2), (3.4) and $(3.9 a, b)$, one finds

$$
L_{u u}^{(x)}(y \rightarrow 0)=\frac{2}{3}+O\left(y^{2 / 3}\right), \quad L_{v v}^{(x)}(y \rightarrow 0) \sim y \quad \text { in SFBL. }
$$

However, over a distance until $L_{u u}^{(x)}$ reaches the homogeneous value of unity $(0.1<$ $y<0.5$, say), the growth rate of $L_{u u}^{(x)}$ is higher. The integral scale of the spanwise velocity $L_{w w}^{(x)}$ exhibits a monotonic decrease.

Profiles of the streamwise integral length scales in USBL are plotted in figure 13. The effect of shear is to increase the streamwise scales of the streamwise and vertical velocity fluctuations, $L_{u u}^{(x)}$ and $L_{v v}^{(x)}$, and to reduce the scale of the spanwise fluctuation, $L_{w w}^{(x)}$, for all heights from the boundary. The increase of $L_{u u}^{(x)}$ indicates elongation with shear of the streamwise extent of the streaks. The near-wall behavior of the integral length scales can be found from (3.5)-(3.8) and (3.10):

$$
\left.\begin{array}{l}
L_{u u}^{(x)}(y \rightarrow 0)=L_{u u}^{(z)}(y \rightarrow 0)=1 / \overline{u^{2}}, \\
L_{v v}^{(x)}(y \rightarrow 0) \sim y / \overline{v^{2}}(1) \\
L_{w w}^{(x)}(y \rightarrow 0)=L_{w w}^{(z)}(y \rightarrow 0)=1 / \overline{w^{2}}
\end{array}\right\} \quad \text { in USBL. }
$$

Near the boundary the scales of the horizontal components decrease with shear, but $L_{v v}^{(x)}$ increases with shear. 


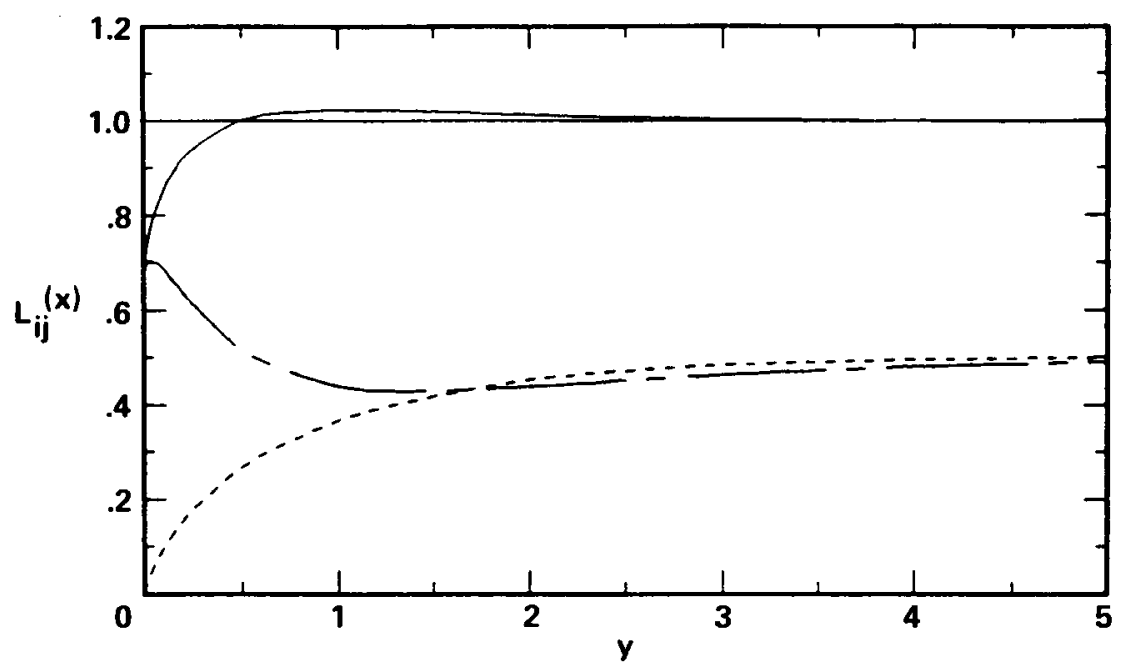

FIgURE 12. Profile of the streamwise integral scales $L_{i j}^{(x)}(y)$ for SFBL $(\beta=0)$. ,$L_{u u}^{(x)}=L_{w w}^{(z)} ;---, L_{v v}^{(x)}=L_{v v}^{(z)} ;---, L_{w w}^{(x)}=L_{u u}^{(z)}$.

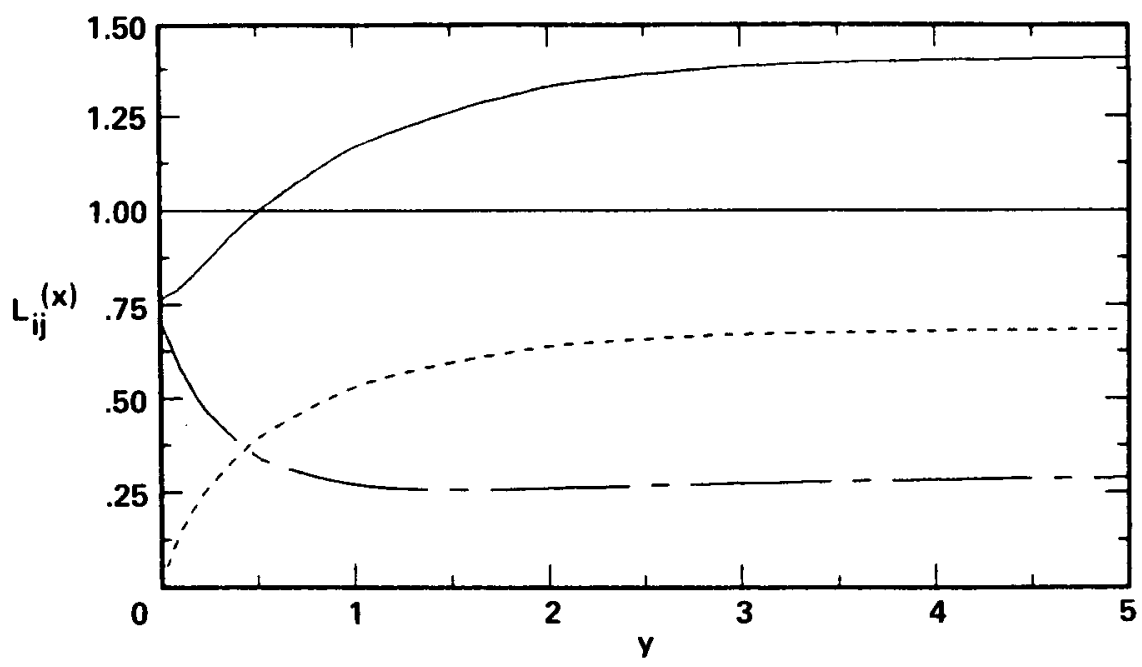

FIgURe 13. Profile of the streamwise integral scales $L_{i j}^{(x)}(y)$ for USBL $(\beta=2)$. ,$L_{u v}^{(x)} ;-\cdots, L_{v v}^{(x)} ;---, L_{w w}^{(x)}$. 


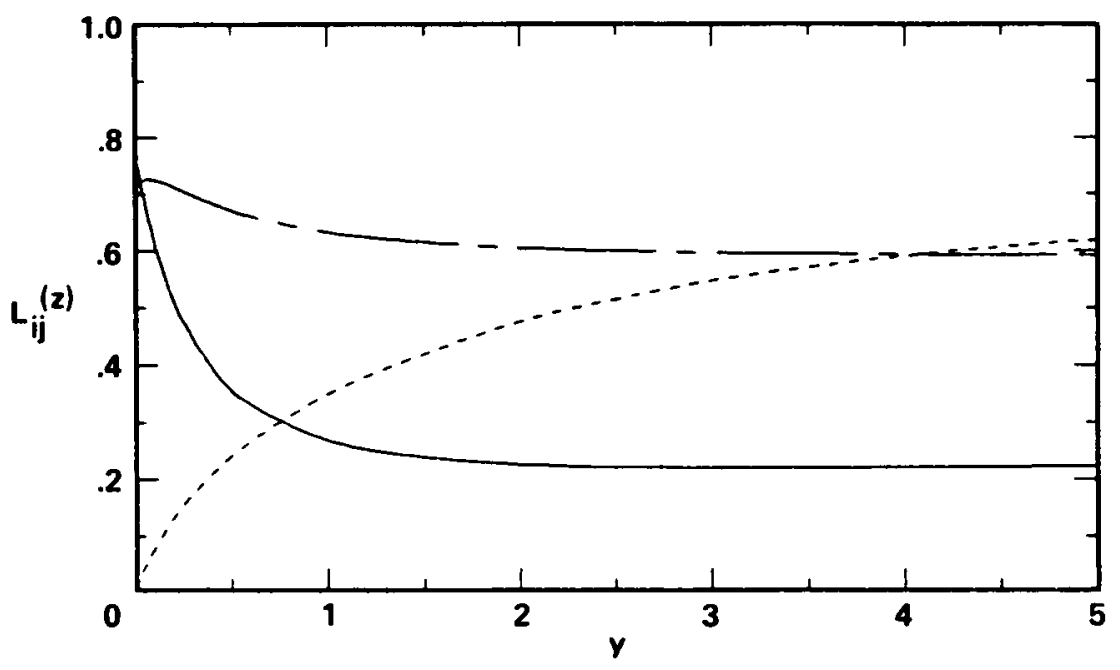

FIGURE 14. Profile of the spanwise integral scales $L_{i j}^{(z)}(y)$ for USBL $(\beta=2)$. — , $L_{u u}^{(z)} ;----, L_{v v}^{(z)} ;---, L_{w w}^{(z)}$.

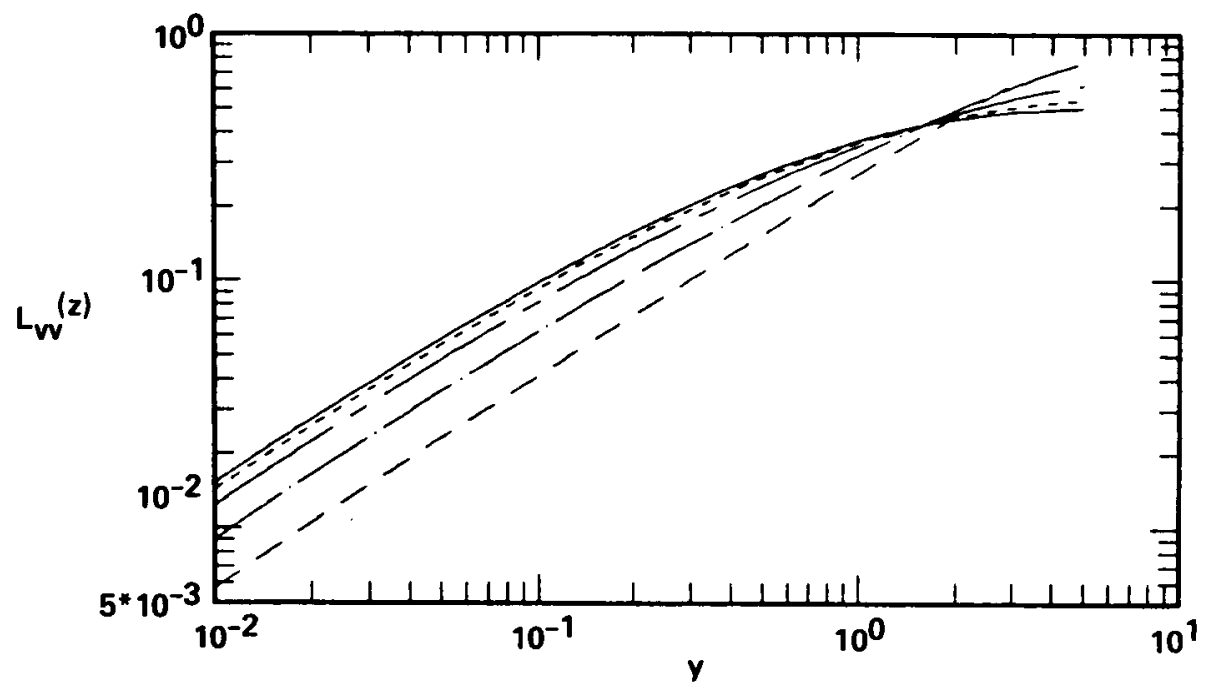

FIGURE 15. Variation with total shear of the profile of $L_{v v}^{(z)} .-, \beta=0 ;-\cdots$, $\beta=1 ;--\longrightarrow, \beta=2 ; \cdots \cdots, \beta=4 ;--\cdots, \beta=8$. 
Figure 14 shows the profiles of the spanwise integral scales in USBL (for comparison with SFBL, see figure 12, where $\left.L_{u u}^{(z)}=L_{w w}^{(x)}, L_{v v}^{(z)}=L_{v v}^{(x)}, L_{w w}^{(z)}=L_{u u}^{(x)}\right)$. For heights away from the boundary, the scales of the streamwise velocity fluctuation, $L_{u u}^{(z)}$, and of the spanwise fluctuation, $L_{w w}^{(z)}$, are markedly decreased with shear, but the scale of the vertical motion, $L_{v v}^{(z)}$, is increased. Near the boundary, however, $L_{u u}^{(z)}$ (and $L_{w w}^{(z)}$ ) increases with shear from the unsheared value $\frac{2}{3}$. This means that since $L_{u u}^{(z)}$ may be interpreted as the characteristic scale for the spanwise extent of the streamwise eddies (streaks), at given total shear the spanwise extent of the streaky eddies widens as the boundary is approached, which is consistent with the reduced bending of vortex near the boundary (see figure $3 b$ ).

We note that $L_{v v}^{(z)}$ is the smallest of all the integral scales in the vicinity of the boundary, and thus it may be a good estimate for the dissipation scale $L_{\epsilon}$. Figure 15 shows the profile of $L_{v v}^{(z)}$ at different values of total shear. The spanwise scale of the vertical fluctuation varies in a self-similar way near the boundary: $L_{v v}^{(z)} \sim A_{\epsilon} y$, where $A_{\epsilon}=A_{\epsilon}(\beta)$ decreases with shear.

\section{Concluding Remarks}

We have studied the effects of shear on turbulence structure near a plane boundary. It has been shown that the blocking of the vertical component of turbulence by the boundary is only slightly affected by the presence of shear (which is consistent with figure 1). However, the shear significantly changes the way in which the boundary affects the horizontal components $\overline{u^{2}}, \overline{w^{2}}$ and the Reynolds stress $-\overline{u v}$. In the SFBL the blocking (or splat) effect leads to the horizontal components being amplified. In a USBL the reduction in $\overline{v^{2}}$ near the boundary reduces the production of $-\overline{u v}$ and $\overline{u^{2}}$ by more than the splat effect increases $\overline{u^{2}}$. The physical explanation is given in $\$ 2$.

We plan to look into other aspects of the surface blocking in sheared turbulence. In order to determine the relative effects of the wall blocking and shear on the vertical length scale, it would be of interest to examine the profiles of the two-point correlations of the vertical component of velocity at different distances from the surface and of the streamwise and vertical components.

A remarkable recent result has been the demonstration that applying the linear (RDT) distortion to an actual realisation of initially homogeneous isotropic velocity fields leads to velocity fields that contain many major large-scale coherent structures (Lee et al. 1987). These structures appear very similar in their scale, shape and distribution to structures that have been found in the direct simulations of homogeneous (uniform) shear flows and in the simulations and experiments of turbulent sheared boundary layers. This similarity implies that RDT is a useful way of computing the structures in shear flows. We intend to study the kinematical properties of the structures in uniform shear over a rigid surface to see how the structures are affected by the blocking effects. 


\section{REFERENCES}

Bertoglio, J. P. 1986 Etude d'une turbulence anisotrope, modélisation de sousmaille et approche statistique. Thése de Doctorat d'État, Université ClaudeBernard Lyon: Lyon, France.

BRADSHAW, P. 1967 'Inactive' motion and pressure fluctuations in turbulent boundary layers. J. Fluid Mech. 30, 241-258.

Durbin, P. A. 1979 Rapid distortion theory of turbulent flows. Ph. D. Thesis, University of Cambridge: Cambridge, England.

Gartshore, I. S., Durbin, P. A. \& HunT, J. C. R. 1983 The production of turbulent stress in a shear flow by irrotational fluctuations. J. Fluid Mech. 137, 307-329.

Hunt, J. C. R. 1973 A theory of turbulent flow round two-dimensional bluff bodies. J. Fluid Mech. 61, 625-706.

HunT, J. C. R. 1984 Turbulence structure in thermal convection and shear-free boundary layers. J. Fluid Mech. 138, 161-184.

Hunt, J. C. R. \& Graham, J. M. R. 1978 Free-stream turbulence near plane boundaries. J. Fluid Mech. 84, 209-235.

Hunt, J. C. R., Moin, P., Moser, R. D. \& Spalart, P. R. 1987a Selfsimilarity of two-point correlations in wall-bounded turbulent flows. Proc. of the 1987 Summer Program, Center for Turbulence Research, Stanford University and NASA-Ames Research Center, pp. 25-36.

Hunt, J. C. R., Spalart, P. R. \& Mansour, N. N. 1987b A general form for the dissipation length scale in turbulent shear flows. Proc. of the 1987 Summer Program, Center for Turbulence Research, Stanford University and NASA-A mes Research Center, pp. 179-184.

Hunt, J. C. R., Stretch, D. D. \& Britter, R. E. 1988 Length scales in stably stratified turbulent flows and their use in turbulence models. In Proc. IMA Conf. on Stably Stratified Flow and Dense Gas Dispersion (ed. J. S. Puttock), Chester, England, April 1986, pp. 285-321, Clarendon Press: Oxford, England.

KIM, J., MoIN, P. \& MOSER, R. D. 1987 Turbulence statistics in fully-developed channel flow at low Reynolds number. J. Fluid Mech. 177, 133-166.

Kline, S. J., Reynolds, W. C., Schraub, F. A. \& Runstadler, P. W. 1967 The structure of turbulent boundary layers. J. Fluid Mech. 30, 741-773.

LEE, M. J., KIM, J. \& MoIN, P. 1987 Turbulence structure at high shear rate. In Sixth Symp. on Turbulent Shear Flows, Toulouse, France, Sept. 7-9, 1987 (ed. F. Durst et al.), pp. 22.6.1-22.6.6.

Lumley, J. L. 1978 Computational modeling of turbulent flows. Adv. Appl. Mech. 18, 123-176.

MAXEY, M. R. 1978 Aspects of unsteady turbulent shear flow, turbulent diffu- 
sion and tidal dispersion. Ph. D. Thesis, University of Cambridge: Cambridge, England.

SPALART, P. R. 1988 Direct simulation of a turbulent boundary layer up to $R_{\theta}=$ 1410. J. Fluid Mech. 187, 61-98.

ThомаS, N. H. \& HANCOCK, P. E. 1977 Grid turbulence near a moving wall. J. Fluid Mech. 82, 481-496.

Townsend, A. A. 1961 Equilibrium layers and wall turbulence. J. Fluid Mech. $11,97-126$.

Townsend, A. A. 1976 The structure of turbulent shear flow. 2nd edn. Cambridge University Press: Cambridge, England.

UzKan, T. \& REYNolds, W. C. 1967 A shear-free turbulent boundary layers. $J$. Fluid Mech. 28, 803-821.

Wong, H. 1987 Turbulence near angled and curved surfaces. Ph. D. Dissertation, University of Cambridge: Cambridge, England. 